\title{
Practical convergence rates for degenerate parabolic equations
}

\author{
K. H. Karlsen and N. H. Risebro and E. B. Storrøsten
}

\section{Introduction}

We are interested in convection-diffusion problems of the form

$$
\left\{\begin{array}{l}
u_{t}+\operatorname{div}(f(u))=\Delta A(u), \quad(t, x) \in(0, T) \times \mathbb{R}^{d}, \\
u(0, x)=u_{0}(x), \\
x \in \mathbb{R}^{d},
\end{array}\right.
$$

where $T$ is some fixed final time and $u$ is the unknown. The initial value $u_{0}$, the convective flux function $f: \mathbb{R} \rightarrow \mathbb{R}^{d}$ and the diffusion function $A: \mathbb{R} \rightarrow \mathbb{R}$ are given functions satisfying

$$
u_{0} \in L^{1}(\mathbb{R}) \cap B V(\mathbb{R}), f, A \text { Lipschitz continuous; } A \text { nondecreasing. }
$$

Whenever $A^{\prime}(u)=0$ for all $u$ in some intervall $[\alpha, \beta] \subset \mathbb{R}$, the equation is referred to as strongly degenerate. Strongly degenerate equations will, in general, possess discontinuous (shock wave) solutions. As such (1) will have to be interpreted in the weak sense. However, as is well known for conservation laws $(A \equiv 0)$, weak solutions are no longer unique and a second condition is needed, the so called en- $^{-}$ tropy condition. More precisely, for $u_{0} \in L^{1} \cap L^{\infty}$, there exists a unique solution $u \in C\left([0, T] ; L^{1}\right) \cap L^{\infty}$ of (1) such that $\partial_{x} A(u) \in L^{2}$ and for all convex functions $S: \mathbb{R} \rightarrow \mathbb{R}$

$$
\partial_{t} S(u)+\operatorname{div} Q_{S}(u)-\Delta R_{S}(u) \leq 0 \text { weakly on }[0, T) \times \mathbb{R}^{d},
$$

where $Q_{S}^{\prime}=f^{\prime} S^{\prime}$ and $R_{S}^{\prime}=A^{\prime} S^{\prime}$. The satisfaction of this inequality for all convex $S$ is the entropy condition, and a weak solution satisfying the entropy condition is

Department of Mathematics, Unversity of Oslo, e-mail: kennethk@math.uio.no, nilshr@ math.uio.no, erlendbs@math.uio.no

This work was supported by the Research Council of Norway (grants no. 250674/F20 and 214495). 
an entropy solution. Entropy solutions are unique, and the initial value problem is well-posed in the class of entropy solutions, see [1]. If $u \mapsto A(u)$ is strictly monotone increasing, then the above entropy condition is not needed to show uniqueness, see the book of Wu and Jin [18]. In several dimensions and for equations with partial diffusivity, one can employ a different entropy solution concept, see [5].

To obtain a finite difference approximation of (1) the flux is discretized by a twopoint monotone numerical flux and the diffusion term is approximated by a second order central difference. As an example, consider the implicit scheme in one spatial dimension and suppose for simplicity that $f^{\prime} \geq 0$. Let $\Delta x>0, \Delta t=T / N$ for $N \geq 1$ and suppose $U=\left\{U_{j}^{n}\right\}_{j \in \mathbb{Z}, 0 \leq n \leq N}$ satisfies

$$
\frac{U_{j}^{n+1}-U_{j}^{n}}{\Delta t}+\frac{f\left(U_{j}^{n+1}\right)-f\left(U_{j-1}^{n+1}\right)}{\Delta x}=\frac{A\left(U_{j+1}^{n+1}\right)-2 A\left(U_{j}^{n+1}\right)+A\left(U_{j-1}^{n+1}\right)}{\Delta x^{2}},
$$

for $0 \leq n<N, j \in \mathbb{Z}$. Set $t_{n}=n \Delta t, x_{j}=j \Delta x$. For simplicity, in this paper, we assume that $f^{\prime}(u) \geq 0$. therefore we use the upwind discretization for the derivative $f(u)_{x}$. In the general case, $f\left(U_{j}\right)$ can be replaced by any monotone two point numerical flux $F\left(U_{j}, U_{j+1}\right)$. The piecewise constant interpolation $u_{\Delta}$ is defined by

$$
u_{\Delta}(t, x)=U_{j}^{n} \text { whenever }(t, x) \in I_{j}^{n}=\left(t_{n-1}, t_{n}\right] \times\left[x_{j-1 / 2}, x_{j+1 / 2}\right) .
$$

In general we let $\Delta t$ be a suitable function of $\Delta x$, i.e., $\Delta t \sim \Delta x^{\gamma}$ for some $\gamma>0$. We are interested in the largest possible $r>0$ for which there exists a constant $C$, independent of $\Delta x$, such that for all $0 \leq t \leq T$

$$
\left\|u_{\Delta}(t, \cdot)-u(t, \cdot)\right\|_{L^{1}} \leq C \Delta x^{r} .
$$

The number $r$ is then referred to as the rate of convergence. Regarding convergence rates we restrict our attention to the $B V$ setting, $u_{0} \in B V$.

In the case of conservation laws $(A \equiv 0)$ it is a classical result due to Kuznetsov that monotone schemes converges with rate $1 / 2$ for general $d \geq 1$ [9], and this is optimal. In general, adding diffusion to the equation yields solutions of higher regularity. One way this extra regularity manifests itself is that if $d=1, x \mapsto A(u(t, x))_{x}$ is of bounded variation for $t>0$. This means that $A(u)$ is continuous, and if $A$ is strictly increasing, so is $u(\cdot, t)$. As the approximation of a derivative by a finite difference is fundamentally dependent on the regularity of the solution, one would expect a higher rate of convergence than in the completely degenerate case $A \equiv 0$. This is indeed the case for parabolic equations, i.e., when $A^{\prime}>\eta$ for some $\eta>0$. Under this assumption, solutions to (1) are smooth, and convergence takes place according to the formal order of the scheme. Thus, naively one would expect a rate for degenerate equations to be no lower than for conservation laws. However, the presence of a term with two derivatives may be "incompatible" with solutions containing discontinuities (belong to $B V$ ) and thereby lower the convergence rate.

Regarding error estimates for weak solutions of degenerate parabolic equations without nonlinear convection, see e.g. [13, 14]. In [4], Ebmeyer considered finite 
element approximations in the case $f \equiv 0$ and $A(u)=u|u|^{m-1}$, and obtained the convergence rate $\left\|u-u_{\Delta x}\right\|_{L^{2}\left((0, T) ; L^{2}(\Omega)\right)} \leq \mathscr{O}\left(\Delta x^{1 / 6}\right)$ independently of $m$. This result holds when $\Omega$ is a bounded domain in $\mathbb{R}^{d}$ with Dirichlet boundary conditions.

Concerning degenerate problems with a nonlinear convective term, it was recently shown in [7] that (6) holds true with $r=1 / 3$ in the case $d=1$. Subsequently, in [8] a semidiscrete scheme (time-continuous) was considered for general $d \geq 1$ using a cartesian grid. Therein, (6) was shown with $r=2 /(19+d)$, for $d>1$ and $r=1 / 3$ for $d=1$. Let us point out that this result holds true for the implicit scheme as well. The discrepancy between naive intuition and the theoretical results, and between $d=1$ and $d>1$ raises the question of whether or not the rates obtained in [7] and [8] are optimal. We remark that the results of [4] are not in conflict with those in $[8,7]$ since the norms are different, and $[4]$ considers weak solutions with Dirichlet conditions on a bounded domain whereas $[8,7]$ treat entropy solutions of the Cauchy problem.

In the purely hyperbolic case Teng and Zhang [17] demonstrated that for monotone schemes, the convergence rate was $r=1$ if the entropy solution consisted of a finite number of shocks. For the general case, Sabac [15] proved that the rate $r=1 / 2$ is optimal for monotone schemes, even for strictly nonlinear flux functions. Also, for strictly convex flux functions, Nessyahu, Tadmor and Tassa [10,11], showed that in a weaker norm, better convergence rates may be obtained. More precisely,

$$
\left\|u-u_{\Delta x}\right\|_{W^{s, p}(\mathbb{R})} \leq \mathscr{O}\left(\Delta x^{(1-s p) / 2 p}\right), \quad-1 \leq s \leq 1 / p .
$$

For $(s, p)=(0,1)$ this recovers the "usual $L^{1}$ rate" of $r=1 / 2$. This result uses that for entropy solutions to scalar conservation laws with a strictly convex flux, $\|u(t, \cdot)\|_{\mathrm{Lip}^{+}} \leq \mathscr{O}(1 / t)$. In the presence of nonlinear diffusion, this does not hold, as simple examples will show. Hence, trying to generalize the results of $[10,11]$ to degenerate parabolic problems does not seem straightforward.

In this paper we seek to shed som light on this question by way of numerical experiments. In most (all but one) of these experiments we have a formula for the exact solution, with isolated discontinuities in the function or its derivative. In all of these cases we find that the actual (numerical) convergence rate is much better than the theoretical rate. To investigate this we use the numerical truncation error.

For more information about monotone schemes for strongly degenerate diffusion equations, we refer to [6], and to [2] for an overview of error estimation theory for conservation laws.

\subsection{Formal rates of convergence.}

Under the assumption of a smooth solution, it is relatively easy to obtain error estimates by considering the truncation error. Due to the low regularity of the entropy solution, it is clear that this does not give a rigorous estimate. Nonetheless, in the 
sequel, several numerical examples are presented where the exact solution is known. In these cases the truncation error may shed some light on the observed rate of convergence. Let us therefore derive a formal error estimate for the scheme (4).

Let $D_{+}, D_{-}$denote the forward and backward difference with respect to $\Delta x$ acting in the $x$-variable respectively. That is, for any function $u=u(x)$

$$
D_{+} u(x)=\frac{u(x+\Delta x)-u(x)}{\Delta x}, \quad D_{-} u(x)=\frac{u(x)-u(x-\Delta x)}{\Delta x} .
$$

The difference quotients $D_{+}^{t}$ and $D_{-}^{t}$ are defined analoguously, but with respect to $\Delta t$ and acting with respect to the $t$-variable. The piecewise constant approximation $u_{\Delta}$, defined by (5), satisfies

$$
D_{-}^{t} u_{\Delta}+D_{-} f\left(u_{\Delta}\right)=D_{-} D_{+} A\left(u_{\Delta}\right), \quad \text { for } 0 \leq t \leq T .
$$

Let $u$ be the entropy solution to (1). The truncation error $\mathscr{T}_{\Delta}$ is defined by

$$
D_{-}^{t} u+D_{-} f(u)=D_{-} D_{+} A(u)+\mathscr{T}_{\Delta}, \quad(\Delta t \leq t \leq T) .
$$

Subtract (8) from (9), multiply by $\operatorname{sign}\left(u-u_{\Delta}\right)$ and integrate to arrive at

$$
\begin{gathered}
\int_{\mathbb{R}} \operatorname{sign}\left(u-u_{\Delta}\right) D_{-}^{t}\left(u-u_{\Delta}\right) d x=-\int_{\mathbb{R}} \\
\operatorname{sign}\left(u-u_{\Delta}\right)\left(\mathscr{A}(u)-\mathscr{A}\left(u_{\Delta}\right)\right) d x \\
+\int_{\mathbb{R}} \operatorname{sign}\left(u-u_{\Delta}\right) \mathscr{T}_{\Delta} d x,
\end{gathered}
$$

where $\mathscr{A}(u)=D_{-}\left(f(u)-D_{+} A(u)\right)$. A fundamental observation regarding the scheme (4) is the fact that $\mathscr{A}$ is an accretive operator, see e.g., [8, Lemma A.1]. This yields

$$
\int_{\mathbb{R}} \operatorname{sign}\left(u-u_{\Delta}\right)\left(\mathscr{A}(u)-\mathscr{A}\left(u_{\Delta}\right)\right) d x \geq 0 .
$$

Let $w=u-u_{\Delta}$ and observe that

$$
D_{-}^{t}|w(t)|=\operatorname{sign}(w(t)) D_{-}^{t} w(t)-\underbrace{\frac{2}{\Delta t} \int_{w(t-\Delta t)}^{w(t)} \delta(z)(z-w(t-\Delta t)) d z}_{\geq 0},
$$

where $\delta(z)$ denotes the Dirac measure centered at $z=0$. Hence, by the above observations

$$
\begin{aligned}
\int_{\mathbb{R}}\left|w\left(t_{n}\right)\right|-\left|w\left(t_{0}\right)\right| d x & =\Delta t \sum_{k=1}^{n} \int_{\mathbb{R}} D_{-}^{t}\left|w\left(t_{k}\right)\right| d x \\
& \leq \Delta t \sum_{k=1}^{n} \int_{\mathbb{R}} \operatorname{sign}\left(w\left(t_{k}\right)\right) D_{-}^{t} w\left(t_{k}\right) d x \\
& \leq \Delta t \sum_{k=1}^{n} \int_{\mathbb{R}} \operatorname{sign}\left(w\left(t_{k}\right)\right) \mathscr{T}_{\Delta}\left(t_{k}\right) d x .
\end{aligned}
$$


Consequently,

$$
\begin{aligned}
\left\|u\left(t_{n}\right)-u_{\Delta}\left(t_{n}\right)\right\|_{L^{1}(\mathbb{R})} \leq & \left\|u_{0}-u_{\Delta}^{0}\right\|_{L^{1}(\mathbb{R})} \\
& +\Delta t \sum_{k=1}^{n} \underbrace{\int_{\mathbb{R}} \operatorname{sign}\left(u\left(t_{k}\right)-u_{\Delta}\left(t_{k}\right)\right) \mathscr{T}_{\Delta}\left(t_{k}\right) d x}_{R_{\Delta}\left(t_{k}\right)}
\end{aligned}
$$

where $u_{\Delta}^{0}$ denotes the initial value of the scheme. Now, let us under the assumption that $u$ is smooth, write out an explicit expression for $\mathscr{T}_{\Delta}$. To this end, subtract (1) from (9) to arrive at

$$
\left(D_{-}^{t} u-\partial_{t} u\right)+\left(D_{-} f(u)-\partial_{x} f(u)\right)=\left(D_{-} D_{+} A(u)-\partial_{x}^{2} A(u)\right)+\mathscr{T}_{\Delta} .
$$

Using Taylor series with remainders we arrive at the expression

$$
\begin{aligned}
\mathscr{T}_{\Delta}(t, x)= & \underbrace{-\frac{1}{\Delta t} \int_{-\Delta t}^{0} \partial_{t}^{2} u(t+\sigma, x)(\sigma+\Delta t) d \sigma}_{\mathscr{T}_{\Delta}^{t}} \\
& \underbrace{-\frac{1}{\Delta x} \int_{-\Delta x}^{0} \partial_{x}^{2} f(u(t, x+\sigma))(\sigma+\Delta x) d \sigma}_{\mathscr{T}_{\Delta}^{f}(t, x)} \\
& \underbrace{+\frac{1}{6 \Delta x^{2}} \int_{0}^{\Delta x} \partial_{x}^{4}(A(u(t, x+\sigma))-A(u(t, x-\sigma)))(\sigma-\Delta x)^{3} d \sigma}_{\mathscr{T}_{\Delta}^{A}(t, x)} .
\end{aligned}
$$

The assumption that $u$ is smooth yields the formal convergence rate of the scheme. Note that the flux-term is of order $\Delta x$, the time-discretization-term is of order $\Delta t$, while the diffusion-term is of order $\Delta x^{2}$. Regarding numerical experiments, it may occur, even with discontinuous solutions, that convergence takes place at the formal order. Let us therefore continue the discussion in order to establish some intuition regarding what happens in the case of an isolated discontinuity in $u$ or its derivatives.

We first restrict our attention to the error associated with the spatial discretization. Let $w$ represent either $f(u(t, \cdot))$ or $A(u(t, \cdot))$, for some fixed $t \in[0, T]$. Assume that $w$ satisfies

$$
w(x)=\left\{\begin{array}{l}
w_{r}(x), x>\xi \\
w_{l}(x), x<\xi
\end{array}\right.
$$

where $\xi$ is a constant, and where both $w_{r}$ and $w_{l}$ belong to $C_{b}^{\infty}(\mathbb{R})$ (the space of infinitely differentiable functions with bounded derivatives). The $k$-th derivative of $w$ satisfies

$$
\partial^{k} w(x)=\sum_{n=0}^{k-1} \underbrace{\left(\partial^{n} w_{r}(\xi)-\partial^{n} w_{l}(\xi)\right)}_{\llbracket \partial^{n} w \rrbracket} \partial^{k-n-1} \delta(x-\xi)
$$




$$
+\underbrace{\mathbb{1}_{x<\xi} \partial^{k} w_{l}(x)+\mathbb{1}_{\xi<x} \partial^{k} w_{r}(x)}_{\mathfrak{S}\left(\partial^{k} w\right)},
$$

in the distributional sense. For the term $\mathscr{T}_{\Delta}^{f}$ this yields

$$
\begin{aligned}
\mathscr{T}_{\Delta}^{f}(\cdot, x)= & \llbracket f(u) \rrbracket \xi \frac{1}{\Delta x} \int_{-\Delta x}^{0} \delta(x+\sigma-\xi) d \sigma \\
& -\llbracket \partial_{x} f(u) \rrbracket \xi \frac{1}{\Delta x} \int_{-\Delta x}^{0} \delta(x+\sigma-\xi)(\sigma+\Delta x) d \sigma \\
& -\frac{1}{\Delta x} \int_{-\Delta x}^{0} \mathfrak{S}\left(\partial_{x}^{2} f(u(\cdot, x+\sigma))\right)(\sigma+\Delta x) d \sigma .
\end{aligned}
$$

Let us split $R_{\Delta}$ defined in (12), according to (14), so that

$$
R_{\Delta}=R_{\Delta}^{t}+R_{\Delta}^{f}+R_{\Delta}^{A} .
$$

By the above, we obtain

$$
R_{\Delta}^{f}=\llbracket f(u) \rrbracket_{\xi} \frac{1}{\Delta x} \int_{\xi}^{\xi+\Delta x} \operatorname{sign}\left(u-u_{\Delta}\right) d x+\mathscr{O}(\Delta x) .
$$

Consider $\mathscr{T}_{\Delta}^{A}$. Inserting the expression for the distributional derivative yields

$$
\begin{aligned}
\mathscr{T}_{\Delta}^{A}(\cdot, x)= & \llbracket \partial_{x} A(u) \rrbracket \xi \frac{1}{\Delta x^{2}} \int_{0}^{\Delta x}(\delta(x+\sigma-\xi)-\delta(x-\sigma-\xi))(\sigma-\Delta x) d \sigma \\
& -\llbracket \partial_{x}^{2} A(u) \rrbracket \xi \frac{1}{3 \Delta x^{2}} \int_{0}^{\Delta x}(\delta(x+\sigma-\xi)-\delta(x-\sigma-\xi))(\sigma-\Delta x)^{2} d \sigma \\
& +\llbracket \partial_{x}^{3} A(u) \rrbracket \xi \frac{1}{6 \Delta x^{2}} \int_{0}^{\Delta x}(\delta(x+\sigma-\xi)-\delta(x-\sigma-\xi))(\sigma-\Delta x)^{3} d \sigma \\
& +\frac{1}{6 \Delta x^{2}} \int_{0}^{\Delta x} \mathfrak{S}\left(\partial_{x}^{4}(A(u(\cdot, x+\sigma))-A(u(\cdot, x-\sigma)))\right)(\sigma-\Delta x)^{3} d \sigma
\end{aligned}
$$

where we applied the fact that $A(u(t, \cdot))$ is continuous. It follows that

$$
\begin{aligned}
R_{\Delta}^{A}=- & \llbracket \partial_{x} A(u) \rrbracket \xi \frac{1}{\Delta x^{2}} \int_{\mathbb{R}} \operatorname{sign}\left(u-u_{\Delta}\right) K_{\Delta}(x, \xi) d x \\
& +\underbrace{\llbracket \partial_{x}^{2} A(u) \rrbracket \xi \frac{1}{3 \Delta x^{2}} \int_{\mathbb{R}} \operatorname{sign}\left(u-u_{\Delta}\right) K_{\Delta}^{2}(x, \xi) d x}_{\mathscr{O}(\Delta x)}+\mathscr{O}\left(\Delta x^{2}\right),
\end{aligned}
$$

where

$$
K_{\Delta}(x, \xi)=\left\{\begin{array}{lr}
x-(\xi-\Delta x) & \text { if } \xi-\Delta x \leq x \leq \xi \\
x-(\xi+\Delta x) & \text { if } \xi \leq x \leq \xi+\Delta x \\
0 & \text { otherwise. }
\end{array}\right.
$$

Let us turn to the error associated with the discretization in time, i.e., $\mathscr{T}_{\Delta}^{t}$. Assume that $u=u(t, x)$ has a discontinuity at $t=\tau(x)$, of the same type as above. Then 


$$
\begin{aligned}
\mathscr{T}_{\Delta}^{t}(t, x)=\llbracket u \rrbracket_{\tau} \frac{1}{\Delta t} \int_{-\Delta t}^{0} \delta(t+\sigma-\tau) d \sigma \\
-\llbracket \partial_{t} u \rrbracket_{\tau} \frac{1}{\Delta t} \int_{-\Delta t}^{0} \delta(t+\sigma-\tau)(\sigma+\Delta t) d \sigma \\
\quad-\frac{1}{\Delta t} \int_{-\Delta t}^{0} \mathfrak{S}\left(\partial_{t}^{2} u(t+\sigma, x)\right)(\sigma+\Delta t) d \sigma,
\end{aligned}
$$

where $\llbracket u \rrbracket_{\tau}=u(\tau+)-u(\tau-)$, using the notation $u(\tau \pm)=\lim _{h \downarrow 0} u(\tau \pm h)$. It follows that

$$
R_{\Delta}^{t}(t)=\frac{1}{\Delta t} \int_{\mathbb{R}} \operatorname{sign}\left(u-u_{\Delta}\right) \llbracket u \rrbracket_{\tau} \mathbb{1}_{t-\Delta t \leq \tau \leq t} d x+\mathscr{O}(\Delta t) .
$$

Suppose the discontinuity lies along an increasing curve $\xi(t)$, so that $\xi(\tau(x))=x$, or equivalently $\tau(\xi(t))=t$. Then

$$
t-\Delta t \leq \tau(x) \leq t \Leftrightarrow \xi(t-\Delta t) \leq x \leq \xi(t),
$$

and so

$$
R_{\Delta}^{t}(t)=\frac{1}{\Delta t} \int_{\xi(t-\Delta t)}^{\xi(t)} \operatorname{sign}\left(u(t, x)-u_{\Delta}(t, x)\right) \llbracket u \rrbracket_{\tau(x)} d x+\mathscr{O}(\Delta t) .
$$

What we now have is a description of how the presence of an isolated discontinuity in the solution will affect the truncation error, possibly causing a slower convergence than the formal order.

\section{Numerical experiments}

Now we investigate the errors for a series of problems where we have an exact solution, or where we have a reference solution which we believe is sufficiently accurate. In the cases where we have a formula for the exact solution, we consider the expressions for the truncation error, and hopefully shed some light on the observed convergence rate.

In addition to the implicit scheme defined by (4), we test the Crank-Nicholson scheme defined by

$$
D_{-}^{t} U_{j}^{n+1}+\frac{1}{2} D_{-}\left(f\left(U_{j}^{n+1}\right)+f\left(U_{j}^{n}\right)\right)=\frac{1}{2} D_{-} D_{+}\left(A\left(U_{j}^{n+1}\right)+A\left(U_{j}^{n}\right)\right) .
$$

For both schemes we set $U_{j}^{0}=\frac{1}{\Delta x} \int_{I_{j}} u_{0}(x) d x$. The Crank-Nicholson scheme is formally second order in time, and if $f \equiv 0$, second order in space as well.

We shall always measure the error as follows. Let $u_{r}$ be some reference solution (either available as an expression, or calculated numerically). The relative error is defined as

$$
E=100 \times \frac{\left\|u_{\Delta}(t, \cdot)-u_{r}(t, \cdot)\right\|_{L^{1}}}{\left\|u_{r}(t, \cdot)\right\|_{L^{1}}} .
$$


In the following examples we set $\Delta t=\Delta x / 2$.

\subsection{No convection}

Set

$$
A(u)=\operatorname{sign}(u) \max (|u|-1,0),
$$

then

$$
u(t, x)=e^{-\pi^{2} t} \cos (\pi x)+\operatorname{sign}(\cos (\pi x))
$$

is an exact solution of

$$
u_{t}=A(u)_{x x} .
$$

Note that $A$ is piecewise linear and continuous, with $A^{\prime}(u)=0$ for $u \in(-1,1)$ and $A^{\prime}(u)=1$ outside this interval. Table 1 shows the errors of the approximate solutions generated by the implicit and Crank-Nicholson schemes for $t=0.2, \Delta x=2 \times 2^{-N}$ with $N=3, \ldots, 12$. We note that for this example, both schemes gave errors which

\begin{tabular}{c|cccccccccc|c}
$N$ & 3 & 4 & 5 & 6 & 7 & 8 & 9 & 10 & 11 & 12 & rate \\
\hline implicit & 7.54 & 4.11 & 2.17 & 1.13 & 0.70 & 0.35 & 0.18 & 0.09 & 0.05 & 0.02 & 0.93 \\
CN & 0.57 & 0.14 & 0.04 & 0.02 & $5 \mathrm{e}-3$ & $1 \mathrm{e}-3$ & $3 \mathrm{e}-4$ & $9 \mathrm{e}-5$ & $2 \mathrm{e}-5$ & $5 \mathrm{e}-6$ & 1.83
\end{tabular}

Table 1 Errors for the implicit and the Crank-Nicholson schemes for (17) - (19).

were consistent with their formal orders, and that the errors produced by the CrankNicholson scheme were significantly smaller than those of the implicit scheme. Figure 1 depicts the exact and the approximate solution using the implicit scheme with $2^{5}$ grid point in the interval $[-1,1]$.

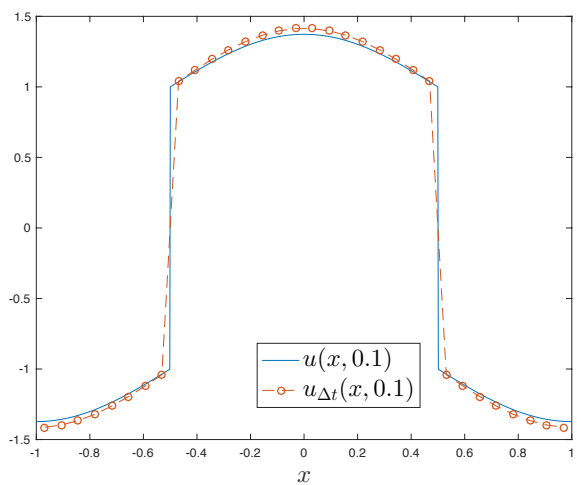

Fig. 1 The exact solution (18) and the approximation found with the implicit scheme with $\Delta x=$ $2^{-4}$ at $t=0.1$ for (17) $-(19)$. 
The fact that convergence takes place according to the formal order follows from the truncation analysis. Consider the truncation error $\mathscr{T}_{\Delta}=\mathscr{T}_{\Delta}^{t}+\mathscr{T}_{\Delta}^{A}$ for the implicit scheme defined in (9). By (14)

$$
\mathscr{T}_{\Delta}^{A}(t, x)=e^{-\pi^{2} t} \frac{\pi^{4}}{6 \Delta x^{2}} \int_{0}^{\Delta x} \underbrace{(\cos (\pi(x+\sigma))-\cos (\pi(x-\sigma)))}_{-2 \sin (\pi x) \sin (\pi \sigma)}(\sigma-\Delta x)^{3} d \sigma,
$$

and so $\mathscr{T}_{\Delta}^{A}(t, x)$ is of order $\Delta x^{3}$. Similarly

$$
\mathscr{T}_{\Delta}^{t}(t, x)=-\frac{\pi^{4}}{\Delta t} e^{-\pi^{2} t} \cos (\pi x) \int_{-\Delta t}^{0} e^{-\pi^{2} \sigma}(\sigma+\Delta t) d \sigma
$$

and so $\mathscr{T}_{\Delta}^{t}(t, x)$ is of the order $\Delta t$. The comparatively large error connected to the time discretization with versus the spatial discretization explains the improved results obtained by the Crank-Nicholson method. A plot of the local truncation error $\operatorname{sign}\left(u-u_{\Delta}\right) \mathscr{T}_{\Delta}$, for $t=0.1$, is given in Figure (2). It is clear that $\mathscr{T}_{\Delta}^{t}$ is the dominant term.

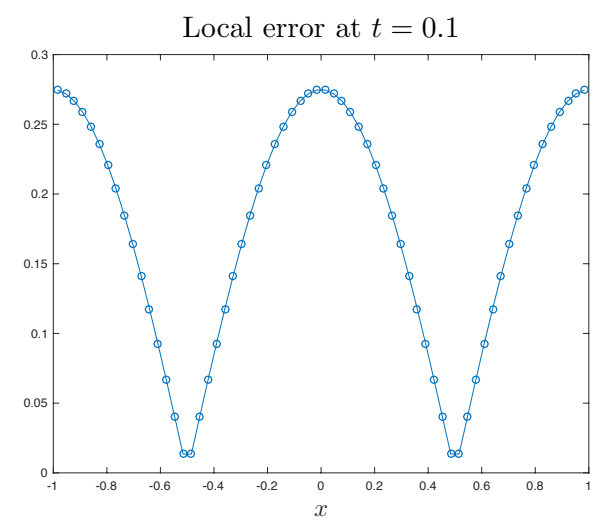

Fig. 2 The truncation error $\operatorname{sign}\left(u-u_{\Delta}\right) \mathscr{T}_{\Delta}$ for the implicit scheme with $\Delta x=2^{-5}$ at $t=0.1$ for (17) - (19).

This convergence can also be explained by the fact that the schemes "do not see" the degenerate diffusion. If we set $\mu=\Delta t / \Delta x^{2}, \omega=4 \mu\left(\cos ^{2}(\pi \Delta x / 2)-1\right)$ and

$$
V_{j}^{n}=(1-\omega)^{-n} \cos (\pi j \Delta x),
$$

then $U_{j}^{n}=V_{j}^{n}+\operatorname{sign}\left(V_{j}^{0}\right)$ will solve the implicit scheme. Similarly, if

$$
W_{j}^{n}=\left(\frac{2+\omega}{2-\omega}\right)^{n} \cos (\pi j \Delta x),
$$


then $W_{j}^{n}+\operatorname{sign}\left(W_{j}^{0}\right)$ is a solution of the Crank-Nicholson scheme. We observe that $\left\{V_{j}^{n}\right\}$ is a solution of the implicit scheme for the linear heat equation $v_{t}=v_{x x}$, hence

$$
\begin{aligned}
\left\|U^{n}-u(n \Delta t, \cdot)\right\|_{L_{1}} & =\left\|V^{m}+\operatorname{sign}\left(V^{0}\right)-e^{-\pi n \Delta t} \cos (\pi \cdot)-\operatorname{sign}(\cos (\pi \cdot))\right\|_{L^{1}(\mathbb{R})} \\
& =\left\|V^{m}-e^{-\pi n \Delta t} \cos (\pi \cdot)\right\|_{L^{1}(\mathbb{R})} \leq \mathscr{O}(\Delta x)
\end{aligned}
$$

Similarly $\left\{W_{j}^{n}\right\}$ is a solution of the Crank-Nicholson scheme for the linear heat equation, thus $\left\|w^{n}-v(n \Delta t, \cdot)\right\|_{L^{1}}=\mathscr{O}\left(\Delta x^{2}\right)$.

\subsection{Linear convection}

If $u$ is given by (18), then $v(t, x)=u(t, x-s t)$ solves the equation

$$
v_{t}+s v_{x}=A(v)_{x x}
$$

where $A$ is given by (17). We use this example with $s=20$, and compute approximate solutions at $t=0.1$, by which time the discontinuities in the initial data will have traveled one period. See Figure 3 for a depiction of the approximate solution.

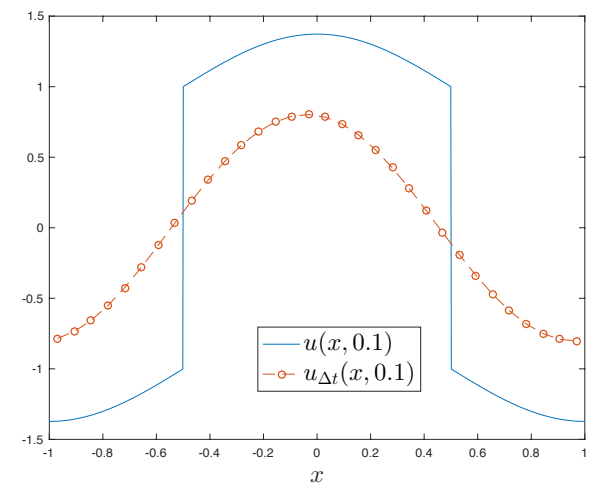

Fig. 3 The exact solution and the approximation found with the implicit scheme with $\Delta x=2^{-4}$ at $t=0.1$ for Example 2.2.

Table 2 shows the errors produced by the implicit and the Crank-Nicholson methods for this example. In this case the errors are comparable for the two methods, and the order has been reduced to about 0.8 .

Since the discontinuities in the solution are moving, they will be detected by the local truncation error, which is shown in Figure 4. Note that the error is much larger, and has two pronounced spikes located at the discontinuities. These spikes may be 


\begin{tabular}{c|cccccccccc|c}
$N$ & 3 & 4 & 5 & 6 & 7 & 8 & 9 & 10 & 11 & 12 & rate \\
\hline implicit & 99.64 & 82.62 & 58.91 & 38.19 & 23.16 & 13.31 & 7.32 & 3.89 & 2.02 & 1.03 & 0.75 \\
CN & 90.77 & 68.91 & 46.24 & 28.77 & 16.88 & 9.44 & 5.09 & 2.66 & 1.37 & 0.69 & 0.80
\end{tabular}

Table 2 Errors for the implicit and the Crank-Nicholson schemes for (20).

explained by the unbounded terms in the truncation error. That is, terms of the order $\frac{1}{\Delta x}$. Note that the solution is discontinuous along $\xi_{k}(t)=x_{k}+s t, x_{k}=k+\frac{1}{2}$ for $k \in$ $\mathbb{Z}$. Then

$$
\begin{aligned}
\mathscr{T}_{\Delta}^{f}(t, x) & =\sum_{k} \llbracket s u \rrbracket_{\xi_{k}} \frac{1}{\Delta x} \mathbb{1}_{\xi_{k}(t) \leq x \leq \xi_{k}(t)+\Delta x}+\mathscr{O}(1), \\
\mathscr{T}_{\Delta}^{t}(t, x) & =\sum_{k} \llbracket u \rrbracket_{\tau_{k}} \frac{1}{\Delta t} \mathbb{1}_{\xi_{k}(t-\Delta t) \leq x \leq \xi_{k}(t)}+\mathscr{O}(1),
\end{aligned}
$$

where $\tau_{k}=\frac{1}{s}\left(x-x_{k}\right)$. The size of the spikes fits very well with the $\mathscr{T}_{\Delta}^{f}$ term. It seems harder to explain the apparently "perfect" change of the sign, yielding a cancellation when integrated in space.

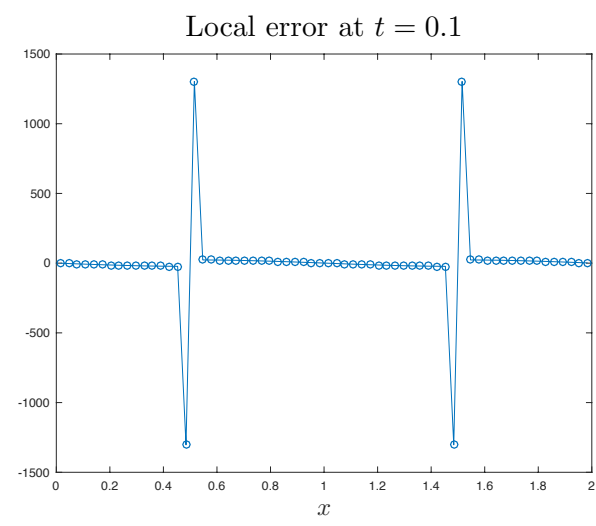

Fig. 4 The truncation error $\operatorname{sign}\left(u-u_{\Delta}\right) \mathscr{T}_{\Delta}$ for the implicit scheme with $\Delta x=2^{-5}$ at $t=0.1$ for Example 2.2.

\subsection{A traveling wave solution}

Define

$$
w(y)= \begin{cases}2\left(1-e^{y / 2}\right) & y \leq 0 \\ 0 & y>0 .\end{cases}
$$

Now $u=w(x-2 t)$ is an entropy solution of

$$
u_{t}+\left(u^{2}\right)_{x}=\left(u^{2}\right)_{x x} .
$$


Observe that in this case $u$ is continuous, and in fact a classical solution except on the line $x=2 t$. We tested this on the interval $x \in[-10,5]$ with $2^{N}$ equally spaced grid points, for $N=3, \ldots, 12$. Here we used Dirichlet boundary conditions, with the exact solution $u(t,-10)$ and $u(t, 5)$ as boundary values. Figure (5) shows the exact and the approximate solution found by the implicit scheme.

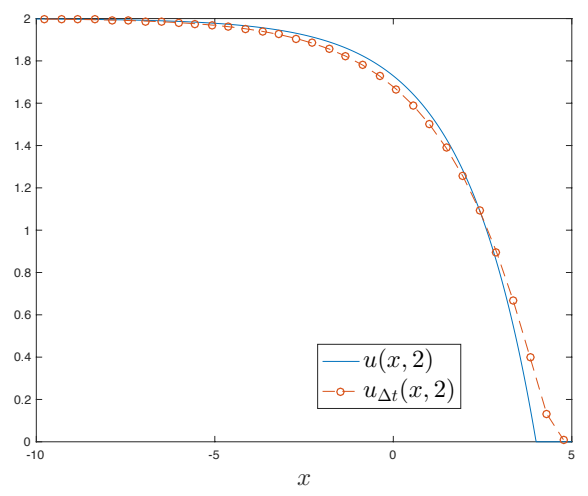

Fig. 5 The exact solution and the approximation found with the implicit scheme with $\Delta x=15 \cdot 2^{-5}$ at $t=2$ for (22).

At $t=2$ the two schemes gave the errors reported in Table 3. For this example,

\begin{tabular}{c|cccccccccc|c}
$N$ & 3 & 4 & 5 & 6 & 7 & 8 & 9 & 10 & 11 & 12 & rate \\
\hline implicit & 8.87 & 5.18 & 2.49 & 1.31 & 0.67 & 0.34 & 0.17 & 0.09 & 0.04 & 0.02 & 0.98 \\
CN & 7.23 & 4.20 & 2.00 & 1.04 & 0.53 & 0.27 & 0.13 & 0.07 & 0.04 & 0.02 & 0.98
\end{tabular}

Table 3 Errors for the implicit and the Crank-Nicholson schemes for (21)

both schemes converge with order 1. In this case, there are no discontinuities, but a kink appears at $x=2 t$. By truncation error analysis, the only possible violation of the formal order convergence could arise due to a jump in $\partial_{x} A(u)$. However,

$$
\partial_{x} A(u(t, x))= \begin{cases}2 w(x-2 t) w^{\prime}(x-2 t) & \text { for } x \leq 2 t, \\ 0, & \text { for } x>2 t,\end{cases}
$$

and so, since $w(0)=0$, there is no jump in $\partial_{x} A(u)$.

\subsection{A moving discontinuity}

The function 


$$
u(t, x)= \begin{cases}2-\exp \left(\frac{1}{4}\left(x-\frac{3}{4} t\right)\right) & , x<\frac{3}{4} t \\ 0, & x \geq \frac{3}{4} t\end{cases}
$$

is an entropy solution to (1) if

$$
f(u)=\left\{\begin{array}{ll}
\frac{1}{2} u^{2}, & u<1, \\
u-\frac{1}{2}, & u \geq 1,
\end{array} \quad A(u)= \begin{cases}0, & u<1, \\
u-1, & u \geq 1 .\end{cases}\right.
$$

We tested this with $2^{N}$ equally spaced grid points in the in interval $[-5,3]$. See Figure 6 for a depiction of the exact and approximate solutions at $t=3$. The errors

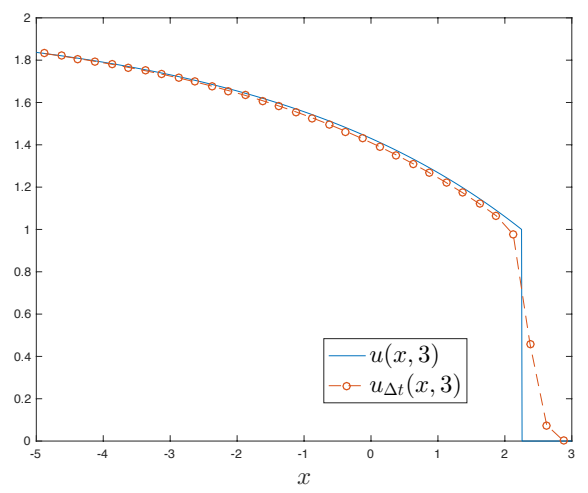

Fig. 6 The exact solution and the approximation found with the implicit scheme with $\Delta x=8 \cdot 2^{-5}$ at $t=3$ with the flux and diffusion given by (24).

at $t=3$ are reported in Table 4 . Again, the convergence takes place according to the formal order of the scheme. In Figure 7 the local error is shown. As expected the truncation error blows up at the discontinuity. As in (2.2) the sign of $\left(u-u_{\Delta}\right)$ seems to change exactly at the spike, making the total error small.

\begin{tabular}{c|cccccccccc|c}
$N$ & 3 & 4 & 5 & 6 & 7 & 8 & 9 & 10 & 11 & 12 & rate \\
\hline implicit & 8.36 & 5.93 & 2.12 & 1.08 & 0.54 & 0.27 & 0.14 & 0.07 & 0.03 & 0.02 & 1.0 \\
CN & 6.22 & 4.49 & 1.33 & 0.67 & 0.33 & 0.17 & 0.08 & 0.04 & 0.02 & 0.01 & 1.0
\end{tabular}

Table 4 Errors for the implicit and Crank-Nicholson schemes with solution (23), and convective and diffuse terms specified by (24).

\subsection{Another moving discontinuity}

Let $v=v(y)$ satisfy the differential equation 


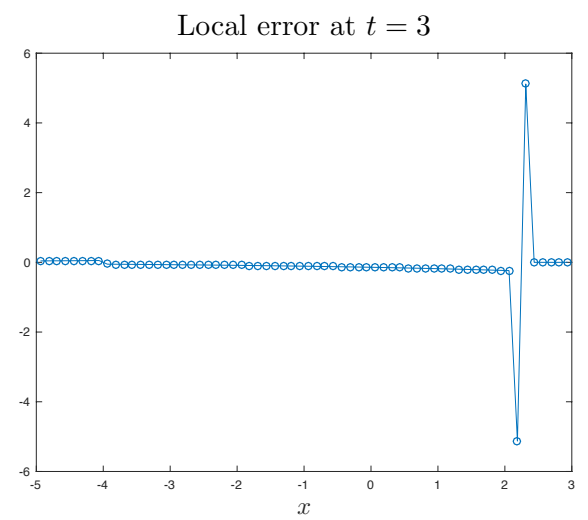

Fig. 7 The truncation error $\operatorname{sign}\left(u-u_{\Delta}\right) \mathscr{T}_{\Delta}$ for the implicit scheme with $\Delta x=2^{-5}$ at $t=3$ for Example 2.4.

$$
\frac{d}{d y}(v-1)^{2}=-u_{l}\left(v-u_{l}\right)+v^{2}-u_{l}^{2}, \quad y<0,
$$

and $v(0-)=1, u_{l}>1$. Remark that this equation can be integrated to yield

$$
v(y)\left(v(y)-u_{l}\right)^{u_{l}-1}=\left(1-u_{l}\right)^{u_{l}-1} e^{\left(u_{l} / 2\right) y},
$$

so if $u_{l}=2$ we get the solution

$$
v(y)=1+\sqrt{1-e^{y}}, \quad y<0 .
$$

Then the function

$$
u(x, t)= \begin{cases}v(x-2 t), & x \leq 2 t \\ 0, & x>2 t\end{cases}
$$

is an entropy solution to the equation

$$
u_{t}+\left(u^{2}\right)_{x}=\left(\chi_{u \geq 1}(u)(u-1)^{2}\right)_{x x} .
$$

We tested the schemes for this example with $x$ in the interval $[-3,3]$ and calculated the solution up to $t=1$. The exact and the approximate solutions are depicted in Figure 8. Table 5 shows the errors in the case. Again we observe that both schemes converge with order 1 , and that there is little difference between the Crank-Nicholson and the implicit scheme. The numerical convergence rate here is close to one for

\begin{tabular}{c|cccccccccc|c}
$N$ & 3 & 4 & 5 & 6 & 7 & 8 & 9 & 10 & 11 & 12 & rate \\
\hline implicit & 7.62 & 6.52 & 2.11 & 1.69 & 0.53 & 0.43 & 0.13 & 0.11 & 0.03 & 0.03 & 1.0 \\
CN & 6.09 & 5.39 & 1.58 & 1.36 & 0.39 & 0.35 & 0.09 & 0.09 & 0.02 & 0.02 & 1.0
\end{tabular}

Table 5 Errors for the implicit and the Crank-Nicholson schemes with solution (26), with solution (25) for $t=1$. 


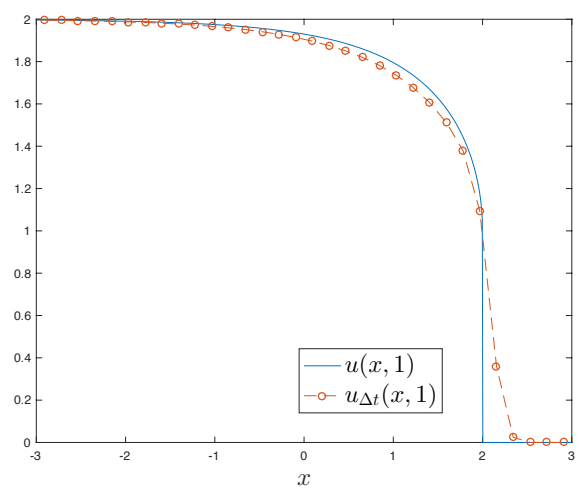

Fig. 8 The exact solution and the approximation found with the implicit scheme with $\Delta x=6 \cdot 2^{-5}$ at $t=1$ for (25) - (26).

both schemes. In Figure 9 we have plotted the local truncation error at $t=1$. We observe that it is much smaller than that of Example 2.2, while it has an oscillatory spike located at the discontinuity $\xi(t)=2 t$. Here, $A(u)$ has a kink, while $u$ and $f(u)$ has a discontinuity. Hence, we expect the truncation error to blow up close to $\xi(t)$. Furthermore,

$$
\partial_{x} f(u(t, x))=\left\{\begin{array}{ll}
2 v(x-2 t) v^{\prime}(x-2 t) & \text { for } x \leq 2 t, \\
0 & \text { for } x>2 t,
\end{array} \quad v^{\prime}(y)=-\frac{e^{y}}{2 \sqrt{1-e^{y}}},\right.
$$

yielding that $\partial_{x} f(u)$ is unbounded as $x \uparrow \xi$. Similarly $\partial_{t} u$ is unbounded as $t \downarrow x / 2$. This could, in comparison with the other examples, explain the apparent asymmetri of the spike. Again, it seems that the change of sign of $u-u_{\Delta}$ is a key factor, preventing the total error from becoming large. In fact, we note that the singularity does not seem to obstruct the convergence much, while this appear hard to explain based on the truncation error.

\subsection{A problem motivated by flow in porous media}

Inspired by models of two-phase flow in porous media, we investigated the convergence rate of our schemes in the case where

$$
f(u)=\frac{u^{2}}{u^{2}+(1-u)^{2}}, \quad A(u)=\frac{1}{10}(f(u))^{2} .
$$

with initial data

$$
u_{0}(x)= \begin{cases}1 & x \in[0.2,1] \\ 0 & \text { otherwise }\end{cases}
$$




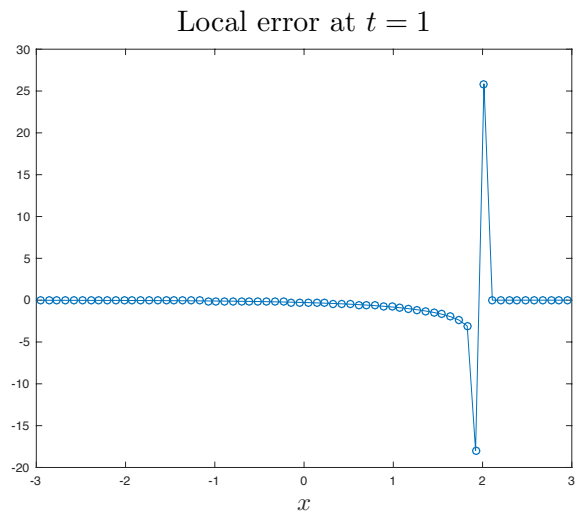

Fig. 9 The truncation truncation error for Example 2.5 at $t=1$ using $2^{6}$ grid points in the interval $[-3,3]$.

Figure 10 shows the Crank-Nicholson approximation $u_{\Delta x}(0.5, \cdot)$ calculated using $2^{9}$ equally spaced grid points in the interval $[0,2]$. For this problem, we have no solution

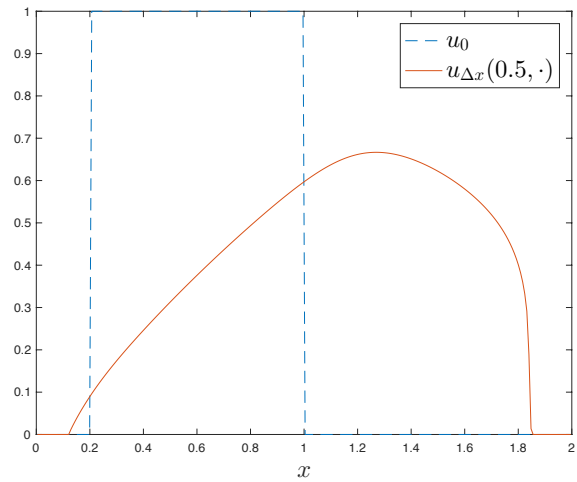

Fig. 10 The approximate solution to (1), with $f$ and $A$ given by (27), initial data given by (28) at $t=0.5$, using $2^{9}$ equally spaced grid points.

on closed form, and to test a convergence rate, we compared our approximations to a reference solution calculated using the Crank-Nicholson scheme on a grid with $2^{12}$ grid points.

Table 6 shows the estimated errors for the two methods applied to this example using $2^{N}$ equally spaced grid points in the interval $[0,2]$. 


\begin{tabular}{c|cccccccc|c}
$N$ & 3 & 4 & 5 & 6 & 7 & 8 & 9 & 10 & rate \\
\hline implicit & 27.8 & 17.8 & 9.4 & 4.9 & 2.7 & 1.4 & 0.6 & 0.2 & 0.97 \\
CN & 27.1 & 17.5 & 9.2 & 4.8 & 2.7 & 1.4 & 0.6 & 0.2 & 0.97
\end{tabular}

Table 6 Errors for the implicit and the Crank-Nicholson schemes with convective and diffuse terms specified by (27).

\subsection{No convection in two dimensions}

We now test the scheme on the two dimensional problem

$$
u_{t}=\Delta A(u),
$$

where $A$ is given by (17). In this case an exact solution is $u(t, x, y)=e^{-\pi^{2} t} v(x, y)+$ $\operatorname{sign}(v(x, y))$, with

$$
v(x, y)=\sin (\pi x)+\sin (\pi y) .
$$

Table 7 shows the error generated by the implicit and the Crank-Nicholson schemes at $t=1 / 4$ on the domain $[-1,1] \times[-1,1]$ and $t=0.2$ with $\Delta x=\Delta y=2^{-N}$ for $N=4, \ldots, 10$. Again, as in the one dimensional case (2.1), convergence takes place

\begin{tabular}{c|ccccccc|c}
$N$ & 4 & 5 & 6 & 7 & 8 & 9 & 10 & rate \\
\hline implicit & 6.24 & 3.05 & 1.51 & 0.75 & 0.37 & 0.18 & 0.09 & 1.0 \\
CN & 0.3872 & 0.0935 & 0.0232 & 0.0058 & 0.0014 & 0.0004 & 0.0001 & 2.0
\end{tabular}

Table 7 Errors for the implicit and the Crank-Nicholson schemes for (29)

according to the formal order of the scheme. As in the one dimensional case, this is not a surprise as $(t, x, y) \mapsto A(u(t, x, y))$ is smooth.

\subsection{Linearly convected discontinuities}

If $u$ denotes the exact solution of the the previous example, then for all constants $a$ and $b, \tilde{u}(t, x, y)=u(t, x-a t, y-b t)$ will solve

$$
\tilde{u}_{t}+a \tilde{u}_{x}+b \tilde{u}_{y}=\Delta A(\tilde{u}),
$$

where $A$ is given by (17). Table 8 shows the errors obtained by the implicit and the Crank-Nicholson methods for this example on the domain $[-1,1] \times[-1,1]$ with $a=\pi$ and $b=1$ and $t=0.2$.

Here, the rate is close to $1 / 2$ which is considerably lower than the formal order. Due to the smoothness of the mapping $(t, x, y) \mapsto A(u(t, x, y))$, the lowered rate is certainly related to the transport rather than the degenerate diffusion. 


\begin{tabular}{c|ccccccc|c}
$N$ & 4 & 5 & 6 & 7 & 8 & 9 & 10 & rate \\
\hline implicit & 39.0 & 28.5 & 18.7 & 12.9 & 8.9 & 6.0 & 4.1 & 0.55 \\
CN & 32.9 & 23.7 & 14.9 & 10.2 & 6.9 & 4.7 & 3.2 & 0.56
\end{tabular}

Table 8 Errors for the implicit and the Crank-Nicholson schemes for (30)

\section{Conclusions}

We have presented a series of numerical experiments aiming at calculating the convergence rate for the implicit (and for the Crank-Nicholson) scheme for nonlinear degenerate convection diffusion equations. In one space dimension, the best known theoretical bound for the convergence rate is $1 / 3$. The technical reason for this rather low bound is the degenerate diffusion, not the non-linear flux term. Our examples do not exhibit this low convergence rate, indeed, in most of them, we record first order convergence or better. However, the examples where we see the "best" convergence rates, are examples where the scheme does not see the degeneracy in the diffusion. These examples have no convective term, and adding a linear convective term reduces the convergence rates to that of the linear convection equation (without diffusive terms). This seems to indicate that the nonlinear degenerate diffusion is not an obstacle to getting the same convergence rate as for scalar conservation laws. Nevertheless, most of our examples compare the numerical approximation with an exact solution on closed form, and we see that there is some cancellation in the local truncation error. The cause of this may be that the data are compatible with the equation, and we would not see this cancellation with other initial data. Unfortunately, it is more difficult to measure errors (in particular truncation errors) when we have no exact solution to compare with. In Section 2.6 we try to do this, and observe first order convergence. Since we have no exact solution, this first order convergence is only self-convergence of the scheme. Also, the nonlinear diffusion in this example is strictly increasing, meaning that the solution is continuous which may not be the worst case scenario.

Unfortunately, we conclude that we lack a complete understanding of the mechanisms leading to the better than theoretical convergence rates observed here, and that whether $1 / 3$ is the optimal rate in one space dimension remains an open problem.

\section{References}

1. José Carrillo, Entropy solutions for nonlinear degenerate problems, Arch. Ration. Mech. Anal. 147 (1999), no. 4, 269-361. MR 1709116 (2000m:35132)

2. Bernardo Cockburn, Continuous dependence and error estimation for viscosity methods, Acta Numer. 12 (2003), 127-180. MR 2249155

3. Bernardo Cockburn and Pierre-Alain Gremaud, A priori error estimates for numerical methods for scalar conservation laws. II. Flux-splitting monotone schemes on irregular Cartesian grids, Math. Comp. 66 (1997), no. 218, 547-572. MR 1408372

4. Carsten Ebmeyer, Error estimates for a class of degenerate parabolic equations, SIAM J. Numer. Anal. 35 (1998), no. 3, 1095-1112 (electronic). MR 1619863 
5. M. Escobedo, J. L. Vázquez, and Enrike Zuazua, Entropy solutions for diffusion-convection equations with partial diffusivity, Trans. Amer. Math. Soc. 343 (1994), no. 2, 829-842. MR 1225573

6. Steinar Evje and Kenneth Hvistendahl Karlsen, Monotone difference approximations of BV solutions to degenerate convection-diffusion equations, SIAM J. Numer. Anal. 37 (2000), no. 6, 1838-1860 (electronic). MR 1766850 (2001g:65110)

7. K. H. Karlsen, N. H. Risebro, and E. B. Storrøsten, $L^{1}$ error estimates for difference approximations of degenerate convection-diffusion equations, Math. Comp. 83 (2014), no. 290, 2717-2762. MR 3246807

8. Kenneth Hvistendahl Karlsen, Nils Henrik Risebro, and Erlend Briseid Storrøsten, On the convergence rate of finite difference methods for degenerate convection-diffusion equations in several space dimensions, ESAIM Math. Model. Numer. Anal. 50 (2016), no. 2, 499-539. MR 3482553

9. N. N. Kuznetsov, The accuracy of certain approximate methods for the computation of weak solutions of a first order quasilinear equation, Ž. Vyčisl. Mat. i Mat. Fiz. 16 (1976), no. 6, 1489-1502, 1627. MR 0483509 (58 \#3510)

10. Haim Nessyahu and Eitan Tadmor, The convergence rate of approximate solutions for nonlinear scalar conservation laws, SIAM J. Numer. Anal. 29 (1992), no. 6, 1505-1519. MR 1191133

11. Haim Nessyahu, Eitan Tadmor, and Tamir Tassa, The convergence rate of Godunov type schemes, SIAM J. Numer. Anal. 31 (1994), no. 1, 1-16. MR 1259963

12. __ The convergence rate of Godunov type schemes, SIAM J. Numer. Anal. 31 (1994), no. 1, 1-16. MR 1259963

13. R. H. Nochetto, Finite element methods for parabolic free boundary problems, Advances in numerical analysis, Vol. I (Lancaster, 1990), Oxford Sci. Publ., Oxford Univ. Press, New York, 1991, pp. 34-95. MR 1138471

14. Florin A. Radu, Iuliu Sorin Pop, and Peter Knabner, Error estimates for a mixed finite element discretization of some degenerate parabolic equations, Numer. Math. 109 (2008), no. 2, 285311. MR 2385655

15. Florin Şabac, The optimal convergence rate of monotone finite difference methods for hyperbolic conservation laws, SIAM J. Numer. Anal. 34 (1997), no. 6, 2306-2318. MR 1480382

16. T. Tang and Zhen Huan Teng, The sharpness of Kuznetsov's $\mathscr{O}(\sqrt{\Delta x}) L^{1}$-error estimate for monotone difference schemes, Math. Comp. 64 (1995), no. 210, 581-589. MR 1270625

17. Zhen-Huan Teng and Ping-wen Zhang, Optimal $L^{1}$-rate of convergence for viscosity method and monotone scheme to piecewise constant solutions with shocks, Advances in numerical mathematics; Proceedings of the Second Japan-China Seminar on Numerical Mathematics (Tokyo, 1994), Lecture Notes Numer. Appl. Anal., vol. 14, Kinokuniya, Tokyo, 1995, pp. 209 225. MR 1469007

18. Zhuoqun Wu, Junning Zhao, Jingxue Yin, and Huilai Li, Nonlinear diffusion equations, World Scientific Publishing Co., Inc., River Edge, NJ, 2001, Translated from the 1996 Chinese original and revised by the authors. MR 1881297 